\title{
Desert Locust Swarms and Child Health
}

\begin{abstract}
This study evaluates how in-utero exposure to an insect pest invasion, particularly, the outbreak of desert locust swarms, affects early childhood health in Africa and Asia over the past three decades (1990-2018). Employing the difference-in-differences model, we find that children being prenatally exposed to the outbreak have their height-for-age, weight-for-height, and weightfor-age z-scores lower by $0.159,0.148$, and 0.155 standard deviations, respectively, compared to unexposed children. Our heterogeneity analyses show that the health setbacks disproportionately fall on children of disadvantaged backgrounds, i.e., those born to lower-educated mothers, poorer mothers, and rural mothers. To the extent that poor health in early life exerts long-lasting irreversible consequences over the life cycle, the study calls for effective measures to minimize the pernicious effects of the desert locust swarm outbreak.
\end{abstract}

